\title{
Combinatorial proofs of some Stirling number formulas
}

\author{
MARK ShatTUCK \\ Department of Mathematics \\ University of Tennessee \\ Knoxville, TN 37996 USA \\ email:shattuck@math.utk.edu
}

(Received: June 29, 2014, and in revised form September 24, 2014.)

\begin{abstract}
In this note, we provide bijective proofs of some recent identities involving Stirling numbers of the second kind, as previously requested. Our arguments also yield generalizations in terms of a well known $q$-Stirling number.
\end{abstract}

Mathematics Subject Classification(2010). 05A19, 11B73.

Keywords: Stirling number, succession, set partition, combinatorial identity.

\section{Introduction}

By a partition of a set, we will mean a collection of pairwise disjoint subsets, called blocks, whose union is the set. For $n \geqslant 1$ and $1 \leqslant k \leqslant n$, let $\mathcal{P}_{n, k}$ denote the set of all partitions of $[n]=\{1,2, \ldots, n\}$ into $k$ blocks and $\mathcal{P}_{n}=\cup_{k} \mathcal{P}_{n, k}$. A member of $\mathcal{P}_{n, k}$ is called a $k$-partition and is said to be in standard form if its blocks $B_{1} / B_{2} / \cdots / B_{k}$ are arranged so that $\min \left(B_{1}\right)<\cdots<\min \left(B_{k}\right)$. Recall that the cardinalities of $\mathcal{P}_{n, k}$ and $\mathcal{P}_{n}$ are given, respectively, by the Stirling number of the second kind $S(n, k)$ and Bell number $B(n)$ (see sequences A008277 and A000110, respectively, in [10] and the recent book [5]). In what follows, if $m$ and $n$ are positive integers, then let $[m, n]=\{m, m+1, \ldots, n\}$ if $m \leqslant n$, with $[m, n]=\varnothing$ if $m>n$. Throughout, the binomial coefficient is given by $\left(\begin{array}{l}n \\ k\end{array}\right)=\frac{n !}{k !(n-k) !}$ if $0 \leqslant k \leqslant n$, with $\left(\begin{array}{l}n \\ k\end{array}\right)$ taken to be zero otherwise.

In [6], several identities involving Stirling and Bell numbers were found by various algebraic methods such as generating functions and use of recurrences. The question of finding combinatorial proofs of the following identities was raised:

$$
\begin{aligned}
& S(n, k)=\sum_{j=0}^{\left\lfloor\frac{n-2}{2}\right\rfloor} \sum_{i=0}^{j}\left(\begin{array}{c}
n-2-j \\
j
\end{array}\right)\left(\begin{array}{c}
j \\
i
\end{array}\right) S(n-2-j-i, k-1) \\
& \quad+\sum_{j=0}^{\left\lfloor\frac{n-1}{2}\right\rfloor} \sum_{i=0}^{j}\left(\begin{array}{c}
n-1-j \\
j
\end{array}\right)\left(\begin{array}{l}
j \\
i
\end{array}\right) S(n-1-j-i, k-1), \quad 1 \leqslant k \leqslant n, \\
& \sum_{j=0}^{\left\lfloor\frac{n-1}{2}\right\rfloor} \sum_{i=0}^{j}\left(\begin{array}{c}
n-1-j \\
j
\end{array}\right)\left(\begin{array}{c}
j \\
i
\end{array}\right) S(n-1-j-i, k-1)=\sum_{j=0}^{n}(-1)^{j} S(n-j, k), \quad 1 \leqslant k \leqslant n,
\end{aligned}
$$

DOI: $10.1515 /$ puma-2015-0009 


$$
\begin{gathered}
\sum_{j=0}^{\left\lfloor\frac{n-1}{2}\right\rfloor} \sum_{i=0}^{j}\left(\begin{array}{c}
n-1-j \\
j
\end{array}\right)\left(\begin{array}{l}
j \\
i
\end{array}\right) B(n-1-j-i)=\sum_{j=0}^{n-1}(-1)^{j} B(n-j), \quad n \geqslant 1, \\
\sum_{j=0}^{\left\lfloor\frac{n-2}{2}\right\rfloor} \sum_{i=0}^{j}\left(\begin{array}{c}
n-2-j \\
j
\end{array}\right)\left(\begin{array}{l}
j \\
i
\end{array}\right) S(n-2-j-i, k-2)=\sum_{j=k}^{n}(-1)^{n-j}\left(\begin{array}{c}
n \\
j
\end{array}\right) S(j, k), \quad 2 \leqslant k \leqslant n .
\end{gathered}
$$

We now recall some terminology from [6]. An ordered pair of elements $(a, b)$ within a subset of $[n]$ that satisfy $b-a \equiv 1(\bmod n)$ is called a (circular) succession. That is, a succession is a pair of consecutive integers or an occurrence of the pair $(n, 1)$. A partition of $[n]$ is said to contain a succession if any of its blocks do. For example, the partition $\pi=15689 / 23 / 47 \in \mathcal{P}_{9,3}$ contains four successions, namely, $(5,6),(8,9),(9,1)$ and $(2,3)$. If a circular succession does not occur as part of a string of consecutive elements of length greater than two (where $n$ and 1 are considered consecutive), then it is said to be detached. In the prior example, only the successions $(5,6)$ and $(2,3)$ of $\pi$ are detached. The question of counting partitions containing a certain number of successions has been considered in various forms; see [7], where linear successions (i.e., those excluding $(n, 1)$ ) were studied, as well as the related papers [3, 8].

Let $c_{r}(n, k)$ denote the cardinality of the subset of $\mathcal{P}_{n, k}$ whose members contain $r$ circular successions. Let $d_{r}(n, k)$ denote the cardinality of the subset of partitions enumerated by $c_{r}(n, k)$ in whose members all of the successions are detached. In [6], it was shown that

$$
\left(\begin{array}{c}
n-r \\
r
\end{array}\right) c_{r}(n, k)=\left(\begin{array}{c}
n-1 \\
r
\end{array}\right) d_{r}(n, k)
$$

It is the purpose of the current note to provide the requested bijective proofs of (1)-(5). Comparable formulas may be given for ordered partitions (that is, those where order matters concerning the blocks themselves) and also in terms of a polynomial generalization of the Stirling numbers, which we consider in the final section.

In the arguments that follow, it will sometimes be more convenient to think of certain sets geometrically. By a linear or circular $n$-tiling, we mean a covering of the numbers $1,2, \ldots, n$, written either in a line or clockwise around a circle, respectively, by indistinguishable squares (pieces covering a single number) and dominos (pieces capable of covering two adjacent numbers). If $0 \leqslant k \leqslant\left\lfloor\frac{n}{2}\right\rfloor$, then let $\mathcal{R}_{n, k}$ and $\mathcal{C}_{n, k}$ denote the sets of linear and circular $n$-tilings containing $k$ dominos. It is well known (see, e.g., [9]) that $\left|\mathcal{R}_{n, k}\right|=\left(\begin{array}{c}n-k \\ k\end{array}\right)$ and $\left|\mathcal{C}_{n, k}\right|=\frac{n}{n-k}\left(\begin{array}{c}n-k \\ k\end{array}\right)$.

\section{Bijective proofs of identities}

We first provide a combinatorial proof of identity (1).

\section{Proof of (1).}

Let us assume $k \geqslant 2$, since the result is clear if $k=1$. Suppose $\pi=B_{1} / B_{2} / \cdots / B_{k} \in \mathcal{P}_{n, k}$ is expressed in standard form. Define, inductively, the sequence $a_{1}<a_{2}<\cdots$ in $[2, n]$ as follows. Let $a_{1}$ be the smallest member of $[2, n]$ occurring in block $B_{1}$ of $\pi$, assuming that there is at least one such 
element (if not, then the sequence is empty). For $\ell>1$, we define $a_{\ell}$ to be the smallest member of $\left[a_{\ell-1}+2, n\right]$ occurring in $B_{1}$. Let us refer to any element of $[2, n]$ equal to $a_{\ell}$ or $a_{\ell}+1$ for some $\ell$ as covered. Note that by definition members of the $a_{\ell}$ sequence must belong to the block $B_{1}$, whereas a covered element of the form $a_{\ell}+1$ may belong to any block.

We now show that $\left(\begin{array}{c}n-1-j \\ j\end{array}\right)\left(\begin{array}{c}j \\ i\end{array}\right) S(n-1-j-i, k-1)$ counts the members of $\mathcal{P}_{n, k}$ having exactly $2 j$ covered elements in $[2, n], j+i$ of which belong to the first block. To do so, first note that there are $\left(\begin{array}{c}n-1-j \\ j\end{array}\right)$ choices for the subset comprising the elements $a_{\ell}, 1 \leqslant \ell \leqslant j$, since such subsets are in oneto-one correspondence with members of $\mathcal{R}_{n-1, j}$, where the numbers $a_{\ell}-1$ correspond to the positions covered by the left halves of dominos within a linear $(n-1)$-tiling. Once the $a_{\ell}$ have been chosen, we select $i$ members from the set $\left\{a_{1}+1, a_{2}+1, \ldots, a_{j}+1\right\}$ to go into a block together with all of the $a_{\ell}$ and the element 1 . Finally, we partition the remaining $n-1-j-i$ elements of $[2, n]$ into $k-1$ blocks, which can be done in $S(n-1-j-i, k-1)$ ways. Putting together all of the blocks gives a member of $\mathcal{P}_{n, k}$ of the desired form, and the process of generating such partitions is seen to be reversible.

For example, let $n=12, k=4, j=3$ and $i=2$, with $a_{1}=3, a_{2}=6$ and $a_{3}=10$, and suppose that 4 and 7 are selected from $\{4,7,11\}$ to go into the first block. If the remaining six elements of [12] are partitioned into three blocks as $\{2,9\},\{5,11,12\},\{8\}$, then one obtains the partition $\{1,3,4,6,7,10\},\{2,9\},\{5,11,12\},\{8\} \in \mathcal{P}_{12,4}$ in which there are six covered elements in [12], five of which belong to the first block.

Summing over all possible $j$ and $i$, where $0 \leqslant i \leqslant j \leqslant\left\lfloor\frac{n-1}{2}\right\rfloor$, implies that the number of members of $\mathcal{P}_{n, k}$ containing an even number of covered elements is given by the second sum on the right-hand side of (1). So it remains to count the members of $\mathcal{P}_{n, k}$ containing an odd number of covered elements. Note that if a partition has an odd number of covered elements, then it must be the case that $n$ is covered and belongs to the first block. Thus counting the remaining partitions is equivalent to counting the number of members of $\mathcal{P}_{n-1, k}$ containing an even number of covered elements, upon adding $n$ to the first block of such partitions. But this is the prior problem with $n$ replaced by $n-1$ and thus gives the first sum on the right-hand side of (1). This completes the enumeration of the members of $\mathcal{P}_{n, k}$ and yields identity (1).

Remark: Summing (1) over $1 \leqslant k \leqslant n$ gives the apparently new Bell number recurrence

$$
\begin{aligned}
B(n)= & \sum_{j=0}^{\left\lfloor\frac{n-2}{2}\right\rfloor} \sum_{i=0}^{j}\left(\begin{array}{c}
n-2-j \\
j
\end{array}\right)\left(\begin{array}{l}
j \\
i
\end{array}\right) B(n-2-j-i) \\
& +\sum_{j=0}^{\left\lfloor\frac{n-1}{2}\right\rfloor} \sum_{i=0}^{j}\left(\begin{array}{c}
n-1-j \\
j
\end{array}\right)\left(\begin{array}{l}
j \\
i
\end{array}\right) B(n-1-j-i), \quad n \geqslant 1 .
\end{aligned}
$$

Formula (6) also follows from allowing any number of blocks in the preceding proof. See [4, 11] for other Bell number recurrences.

\section{Proofs of (2) and (3).}

We prove (2). Allowing the number of blocks to vary within a partition in the argument that follows will give (3). Let us assume $k \geqslant 2$ in (2), since the result is clear if $k=1$. Let $\mathcal{A}_{n, k}=\cup_{j=0}^{n} \mathcal{P}_{n-j, k}$ 
and $\pi \in \mathcal{P}_{n-j, k}$ have sign $(-1)^{j}$. Then the right-hand side of (2) gives the sum of the signs of all the members of $\mathcal{A}_{n, k}$.

We define a sign-reversing involution on $\mathcal{A}_{n, k}$ as follows. Consider whether the element $m$ within $\pi \in \mathcal{P}_{m, k}$, where $k \leqslant m \leqslant n$, belongs to a block of $\pi$ as part of a string of consecutive elements of even or odd length, that is, the parity of $t$ such that $m, m-1, \ldots, m-t+1$ belong to the same block but $m-t$ does not. If $t$ is even, then we delete the element $m$ from $\pi$ and if $t$ is odd, then we add the element $m+1$ to $\pi$. For example, if $n=9, k=3$ and $m=7$, then $\pi=\{1,3\},\{2,5,6,7\},\{4\} \in \mathcal{P}_{7,3}$ would map to $\pi^{\prime}=\{1,3\},\{2,5,6,7,8\},\{4\} \in \mathcal{P}_{8,3}$.

This operation is seen to be a sign-reversing involution of $\mathcal{A}_{n, k}$ that is not defined for $\pi \in \mathcal{P}_{n, k}$ such that $n$ belongs to a block as part of a string of consecutive elements of odd length. Note that all members of this subset of $\mathcal{A}_{n, k}$ have positive sign, which we'll denote by $\mathcal{A}_{n, k}^{*}$. To complete the proof of (2), we need to show that the left-hand side gives the cardinality of $\mathcal{A}_{n, k}^{*}$.

By the proof of (1) above, the left-hand side of (2) counts all members of $\mathcal{P}_{n+1, k}$ containing an odd number of covered elements, of which $n+1$ must be the largest and hence belongs to the same block as 1 . In addition, there would also be a possibly empty string of the form $n, n-1, \ldots, n-(2 t-1)$ comprising part of this block. Hence, deleting 1 from all such partitions and subtracting one from each element of $[2, n+1]$ results in the members of $\mathcal{A}_{n, k}^{*}$ and completes the proof of (2).

\section{Proof of (4).}

Suppose $n$ and $k$ are given and that $k \leqslant j \leqslant n$. Consider the set of ordered pairs $(S, \rho)$, where $S$ is a subset of $[n]$ of size $n-j$ and $\rho$ is a partition of the set $[n]-S$ having $k$ blocks. Let $\mathcal{B}_{n, j}$ denote the set of all such ordered pairs $(S, \rho)$ and $\mathcal{B}_{n}=\cup_{j=k}^{n} \mathcal{B}_{n, j}$. Let members of $\mathcal{B}_{n, j}$ have sign $(-1)^{n-j}$. Then the right-hand side of (4) gives the sum of the signs of all members of $\mathcal{B}_{n}$.

We define a sign-reversing involution on $\mathcal{B}_{n}$ as follows. Given $(S, \rho) \in \mathcal{B}_{n}$, let $m$ be the smallest element of $[n]-S$ and let $s$ be the smallest element in $[m+1, n]$ satisfying either (a) $s \in S$ or (b) $s \in[n]-S$, with $s$ belonging to the same block of $\rho$ as $m$ does. Assuming $s$ exists, we define an involution on $\mathcal{B}_{n}$ by moving $s$ from $S$ to the block containing $m$ in $\rho$ if (a) occurs or by moving $s$ from $\rho$ to the set $S$ if (b) occurs. Note that this operation defines an involution which changes the sign but preserves both $m$ and $s$. For example, if $n=10, k=3, j=7, S=\{1,4,8\}$ and $\rho=\{2,5,10\},\{3,9\},\{6,7\}$, then $m=2$ and $s=4$ and $(S, \rho)$ would be paired with $\left(S^{\prime}, \rho^{\prime}\right)$, where $S^{\prime}=\{1,8\}$ and $\rho^{\prime}=\{2,4,5,10\},\{3,9\},\{6,7\}$.

This operation is not defined for $(S, \rho) \in \mathcal{B}_{n}$ in which $S$ is of the form $[\ell]$ for some $0 \leqslant \ell \leqslant n-k$, with the smallest element of $[n]-S$ in a block by itself in $\rho$. Upon deleting the element $m$ (and the block containing it), we see that the set of survivors of the involution is equivalent to the set $\mathcal{A}_{n-1, k-1}=\cup_{j=k-1}^{n-1} \mathcal{P}_{j, k-1}$ from the preceding proof of $(2)$, where the sign of $\lambda \in \mathcal{P}_{j, k-1}$ is now given by $(-1)^{n-1-j}$. Applying now the involution used in the proof of (2) to this set of survivors implies that the signed sum of all members of $\mathcal{B}_{n}$ equals the cardinality of the set $\mathcal{A}_{n-1, k-1}^{*}$ from that proof. Note that $\left|\mathcal{A}_{n-1, k-1}^{*}\right|$ is given by the left-hand side of (2), with $n$ replaced by $n-1$ and $k$ by $k-1$, which completes the proof of (4).

\section{Proof of (5).}


We prove (5) in the slightly rewritten form

$$
\frac{n}{n-r}\left(\begin{array}{c}
n-r \\
r
\end{array}\right) c_{r}(n, k)=\left(\begin{array}{l}
n \\
r
\end{array}\right) d_{r}(n, k) .
$$

Consider the set $\mathcal{U}$ of ordered pairs $(\alpha, \pi)$, where $\alpha \in \mathcal{C}_{n, r}$ and $\pi$ is a member of $\mathcal{P}_{n, k}$ having $r$ circular successions. Let $\mathcal{V}$ denote the set of ordered pairs $\left(\beta, \pi^{\prime}\right)$, where $\beta$ is a subset of $[n]$ of size $r$ and $\pi^{\prime}$ is a member of $\mathcal{P}_{n, k}$ having $r$ circular successions all of which are detached.

We'll define a bijection $f$ between $\mathcal{U}$ and $\mathcal{V}$, which will imply $(7)$. Let $(\alpha, \pi) \in \mathcal{U}$. We first consider $i \in[n]$ such that within $\pi$ the letter $i-1$ occurs in the same block as $i$ if $i>1$, along with 1 if $n$ occurs in the same block as 1 . We take these elements and form a set which we will call $\beta$. We then take the remaining $n-r$ elements of $\pi$ within their respective blocks and reduce them to letters in $[n-r]$ to obtain a member $\widehat{\pi}$ of $\mathcal{P}_{n-r, k}$ containing no circular successions.

First suppose that it is not the case that there is a domino covering the numbers $n$ and 1 in $\alpha$. Let $i_{1}<i_{2}<\cdots<i_{r}$ denote the members of $[n]$ that are covered by the right halves of dominos in $\alpha$ when traversing the circle clockwise. We first add the element $i_{1}$ to the block of $\widehat{\pi}$ which contains $i_{1}-1$, increasing all members of $\left[i_{1}, n-r\right]$ in $\widehat{\pi}$ by one. To the resulting partition of $[n-r+1]$, we add the element $i_{2}$ to the block containing $i_{2}-1$ and increase all members of $\left[i_{2}, n-r+1\right]$ by one. Repeat for each subsequent element until all of the $i_{j}$ have been added. Note that in the final partition, the elements $n$ and 1 do not occur together in the same block since $n-r$ and 1 did not occur in the same block of $\widehat{\pi}$.

If there is a domino covering $n$ and 1 in $\alpha$, then we repeat the above procedure, this time letting $i_{1}<i_{2}<\cdots<i_{r-1}$ denote the members of $[2, n-1]$ that are covered by the right halves of dominos. To the resulting member of $\mathcal{P}_{n-1, k}$, we add $n$ to the block containing 1 .

In either case, let $\pi^{\prime}$ denote the member of $\mathcal{P}_{n, k}$ obtained from $\widehat{\pi}$. Note that $\pi^{\prime}$ contains no strings of consecutive letters of length greater than two since $\widehat{\pi}$ contains no circular successions and since $i_{j+1}-i_{j}>1$ for all $j$. This implies $\pi^{\prime}$ contains $r$ circular successions, all of which are detached. Let $f(\alpha, \pi)=\left(\beta, \pi^{\prime}\right)$. The above procedure for generating $\left(\beta, \pi^{\prime}\right)$ from $(\alpha, \pi)$ is seen to be reversible, upon considering cases whether or not 1 and $n$ belong to the same block of $\pi^{\prime}$. Note that the larger numbers in the detached successions of $\pi^{\prime}$ correspond to the elements of the $i_{j}$ sequence, which determines $\alpha$ and can in turn be used to recreate $\widehat{\pi}$ and $\pi$ given $\beta$. Thus $f$ yields the desired bijection between $\mathcal{U}$ and $\mathcal{V}$.

To illustrate the bijection $f$, suppose $n=9, k=4$ and $r=3$. Let $(\alpha, \pi) \in \mathcal{U}$ be given by $\alpha=d s d s^{2} d \in \mathcal{C}_{9,3}$ (where this sequence represents the order of the pieces encountered as one traverses the circle clockwise, starting with 1 , where 1 and 2 are covered by the same domino) and $\pi=1389 / 24 / 56 / 7 \in \mathcal{P}_{9,4}$. Then $\beta=\{1,6,9\}$ and $\widehat{\pi}=13 / 26 / 4 / 5 \in \mathcal{P}_{6,4}$, with $i_{1}=2, i_{2}=5$ and $i_{3}=9$. Inserting the $i_{j}$ sequentially into $\widehat{\pi}$ as described above gives

$$
\widehat{\pi}=13 / 26 / 4 / 5 \rightarrow 124 / 37 / 5 / 6 \rightarrow 1245 / 38 / 6 / 7 \rightarrow \pi^{\prime}=1245 / 389 / 6 / 7 \in \mathcal{P}_{9,4} .
$$

Note that $\pi^{\prime}$ contains three (detached) circular successions, which implies $\left(\beta, \pi^{\prime}\right) \in \mathcal{V}$.

\section{Generalizations}

Let $\sigma(n, k)$ denote the number of partitions of $[n]$ into $k$ blocks where the order of the blocks matters. Equivalently, the number $\sigma(n, k)$ counts the set of surjective functions from $[n]$ to $[k]$. Note that 
$\sigma(n, k)=k ! S(n, k)$; see sequence A019538 in OEIS [10] for further information on these numbers. Upon multiplying both sides by $k$ !, the identities (1), (2) and (4) above are seen to give comparable formulas for $\sigma(n, k)$ since the second argument of any Stirling numbers appearing in the sums is fixed. For example, from (4), we get

$$
k(k-1) \sum_{j=0}^{\left\lfloor\frac{n-2}{2}\right\rfloor} \sum_{i=0}^{j}\left(\begin{array}{c}
n-2-j \\
j
\end{array}\right)\left(\begin{array}{l}
j \\
i
\end{array}\right) \sigma(n-2-j-i, k-2)=\sum_{j=k}^{n}(-1)^{n-j}\left(\begin{array}{l}
n \\
j
\end{array}\right) \sigma(j, k) .
$$

It is also possible to generalize the previous identities in terms of a well known $q$-generalization of the Stirling numbers. Let $n_{q}=1+q+\cdots+q^{n-1}$ if $n \geqslant 1$, with $0_{q}=0$. Carlitz [1] considered the $q$-Stirling numbers $S_{q}(n, k)$ defined by the recurrence

$$
S_{q}(n, k)=S_{q}(n-1, k-1)+k_{q} S_{q}(n-1, k), \quad n, k \geqslant 1,
$$

with $S_{q}(0, k)=\delta_{k, 0}$ for $k \geqslant 0$, and showed that they are given explicitly by

$$
S_{q}(n, k)=\frac{1}{q^{\left(\begin{array}{c}
k \\
2
\end{array}\right)} k_{q} !} \sum_{j=0}^{k}(-1)^{j} q^{\left(\begin{array}{c}
j \\
2
\end{array}\right)}\left(\begin{array}{l}
k \\
j
\end{array}\right)_{q}\left[(k-j)_{q}\right]^{n},
$$

where $k_{q} !=\prod_{i=1}^{k} i_{q}$ and $\left(\begin{array}{l}k \\ j\end{array}\right)_{q}=\frac{k_{q} !}{j_{q} !(k-j)_{q} !}$. Later, Carlitz [2] defined a statistic on $\mathcal{P}_{n, k}$ for which $S_{q}(n, k)$ is its distribution polynomial. We'll denote it here by $\widetilde{w}$ as in [12]. If $\pi=B_{1} / B_{2} / \cdots / B_{k} \in \mathcal{P}_{n, k}$ is in standard form, then let $\widetilde{w}(\pi)=\sum_{i=1}^{k}(i-1)\left(\left|B_{i}\right|-1\right)$. It is well known and can be shown using the recurrence that

$$
S_{q}(n, k)=\sum_{\pi \in \mathcal{P}_{n, k}} q^{\widetilde{w}(\pi)}
$$

Using this interpretation for $S_{q}(n, k)$, one may generalize identities (1) and (2) as follows for $1 \leqslant k \leqslant n$ :

$$
\begin{aligned}
S_{q}(n, k)= & \sum_{j=0}^{\left\lfloor\frac{n-2}{2}\right\rfloor} \sum_{i=0}^{j}\left(\begin{array}{c}
n-2-j \\
j
\end{array}\right)\left(\begin{array}{c}
j \\
i
\end{array}\right) q^{n-1-k-j-i} S_{q}(n-2-j-i, k-1) \\
& +\sum_{j=0}^{\left\lfloor\frac{n-1}{2}\right\rfloor} \sum_{i=0}^{j}\left(\begin{array}{c}
n-1-j \\
j
\end{array}\right)\left(\begin{array}{c}
j \\
i
\end{array}\right) q^{n-k-j-i} S_{q}(n-1-j-i, k-1)
\end{aligned}
$$

and

$$
\sum_{j=0}^{\left\lfloor\frac{n-1}{2}\right\rfloor} \sum_{i=0}^{j}\left(\begin{array}{c}
n-1-j \\
j
\end{array}\right)\left(\begin{array}{l}
j \\
i
\end{array}\right) q^{n-k-j-i} S_{q}(n-1-j-i, k-1)=\sum_{j=0}^{n}(-1)^{j} S_{q}(n-j, k) .
$$

Weighted versions of the preceding combinatorial arguments apply to these formulas. Note that the $q^{n-1-k-j-i}$ factor in the first sum on the right-hand side of $(9)$ accounts for the fact that within the enumerated class of partitions the $n-2-j-i$ elements occurring in blocks beyond $B_{1}$, excluding the smallest elements within each of these blocks, contribute one more towards the $\widetilde{w}$ value than they 
ordinarily would if they comprised a partition by themselves. The $q^{n-k-j-i}$ factor in the second sum may be accounted for similarly.

To explain (10), it is more convenient to replace each $i \in[n]$ with $n+1-i$ and assume that the blocks are arranged in decreasing order of largest elements when applying the $\widetilde{w}$ statistic. Note that the involution used in the proof of (2) above reverses the sign, but does not change the weight, since the element that is either added or taken away belongs to the first block and hence does not affect the $\widetilde{w}$ value. The weight factor of $q^{n-k-j-i}$ appearing in the sum on the left-hand side of 10 arises for much the same reason as before since $j+i$ other elements belong to the same block as $n$ within the enumerated class of partitions.

\section{References}

[1] L. Carlitz, q-Bernoulli numbers and polynomials, Duke Math. J., 15 (1948) 987-1000.

[2] L. Carlitz, Generalized Stirling numbers, Combinatorial Analysis Notes, Duke University, (1968) 8-15.

[3] W. Chu And C. Wei, Set partitions with restrictions, Discrete Math., 308 (2008) 3163-3168.

[4] H. W. Gould and J. Quaintance, Implications of Spivey's Bell number formula, J. Integer Seq., 11 (2008) Art. 08.3.7.

[5] T. Mansour, Combinatorics of Set Partitions, Chapman \& Hall/CRC, Boca Raton, Florida, 2012.

[6] T. Mansour and A. O. Munagi, Set partitions with circular successions, European J. Combin., $42(2014)$ 207-216.

[7] A. O. Munagi, Extended set partitions with successions, European J. Combin., 29 (2008) $1298-1308$.

[8] M. ShatTuck, Recounting relations for set partitions with restrictions, Ars Combin., 91 (2009) 267-270.

[9] M. A. Shattuck And C. G. Wagner, Parity theorems for statistics on domino arrangements, Electron. J. Combin., 12 (2005) \#N10.

[10] N. J. Sloane, The On-Line Encyclopedia of Integer Sequences, http://oeis.org/.

[11] M. Z. SpIVEy, A generalized recurrence for Bell numbers, J. Integer Seq., 11 (2008) Art. 08.2.5.

[12] C. G. Wagner, Partition statistics and q-Bell numbers $(q=-1), \quad$ J. Integer Seq., 7 (2004) Art. 04.1.1. 